J.-L. COLLIAT

ELF Exploration Production avenue Larribau 64018 Pau Cedex

\title{
Caissons à succion pour l'ancrage de structures pétrolières en mer profonde
}

Un nouveau type d'ancre, composé de caissons mis en place par succion, connaít actuellement un intérêt important dans l'industrie pétrolière, notamment pour les grandes profondeurs d'eau, du fait d'une plus grande fiabilité, de leur capacité à reprendre des efforts inclinés avec des lignes d'amarrage tendues et d'une réduction des moyens d'installation requis. Une des premières applications a été faite par ELF Congo en 1995, pour l'ancrage d'une barge de production sur le champ de Nkossa par 200 mètres de profondeur d'eau. Les 12 caissons à succion en acier, avec un diamètre de 5 mètres et une hauteur de 13 mètres, ont été installés avec succès dans des argiles molles normalement consolidées, représentatives des sites par grands fonds. Les hypothèses générales du dimensionnement sont données, ainsi que le résultat des opérations de mise en place sur le site et l'évaluation du comportement des caissons enregistré en cours d'installation. Les résultats obtenus, en parfait accord avec les prévisions, ont démontré la fiabilité de la procédure d'installation. Une nouvelle application pour le Groupe ELF aura lieu début 2001 en Angola sur le champ de Girassol par 1400 mètres de profondeur d'eau. Dans ce cas, les 16 caissons, de diamètre 4,5 mètres et d'une hauteur de 17 mètres, serviront à I'ancrage d'un FPSO sur un des champs pétroliers les plus profonds au monde.

\section{Suction caissons for anchoring oil production structures at deep water sites}

A new type of anchor, composed of suction caissons, presently finds a strong interest in the offshore oil industry, in particular for deep waters, because of their improved reliability, their vertical loading capacity in taut leg moorings, and the reciuced installation equipment required. One of the first applications has been made by ELF Congo in 1995, for anchoring a process barge at the Nkossa field in about 200 metres of water depth. The 12 steel suction caissons, 5 metres in diameter and 13 metres high, were successfully installed in soft normally consolidated clays, representative of deep water sites. The general design assumptions are given in the paper, together with the results of the installation operations with the evaluation of the monitored installation performance. The results obtained were in perfect agreement with the predictions, thus demonstrating the reliability of the design installation procedure. A new application for the ELF Group will be made early 2001 in Angola at the Girassol field in 1.400 metres of water depth. In that case, the 16 steel suction caissons, with a diameter of 4.50 metres and 17 metres high, will be used for anchoring an FPSO at one of the deepest oil fields world wide to date. 


\section{Introduction}

L'industrie pétrolière s'engage dans des profondeurs d'eau de plus en plus grandes, notamment dans le golfe du Mexique, en mer du Nord, au large du Brésil et dans le golfe de Guinée. Vers 1980, au début des interventions par robot télé-opéré ( $\propto$ ROV $»$ Remote Operated Vehicle), les « grands fonds » débutaient vers 200 mètres de profondeur d'eau, soit plus ou moins à la limite des interventions par plongée sous-marine, cette limite étant ensuite portée à environ 500 mètres une dizaine d'années plus tard. Actuellement, des forages d'exploration sont réalisés par plus de 2000 mètres d'eau (avec, par exemple, la récente découverte par ELF Exploration Inc. du champ d'Aconcagua par 2150 mètres de profondeur dans le golfe du Mexique) et des champs pétroliers sont mis en production dans des profondeurs comprises entre 1000 et 1850 mètres (record actuel de la tête de puits sousmarine du champ de Roncador au large du Brésil).

Le nouveau terme " ultra grand fond $)$ est maintenant utilisé pour la tranche d'eau comprise entre 1500 et 3000 mètres, ceci étant considéré comme une limite de faisabilité avec les technologies actuelles. Les spécialistes en géotechnique marine ont suivi ce mouvement et ont su innover pour répondre aux nouveaux besoins ainsi créés [1].

Dans les grandes profondeurs d'eau (> 500 mètres), de nouveaux concepts de structures de production sont considérés, avec le double souci constant d'une amélioration de la fiabilité et d'une maîtrise des coûts de développement. Les différentes structures pétroliẽres flottantes sont données à la figure 1 : plate-forme à lignes tendues ( $₫$ TLP ) Tension Leg Platform), bouée flottante «SPAR », unité flottante de production et stockage ( FPSO $)$ Floating Production Storage and Offloading) et unité semi-submersible de production ( $($ FPU ») Floating Production Unit).

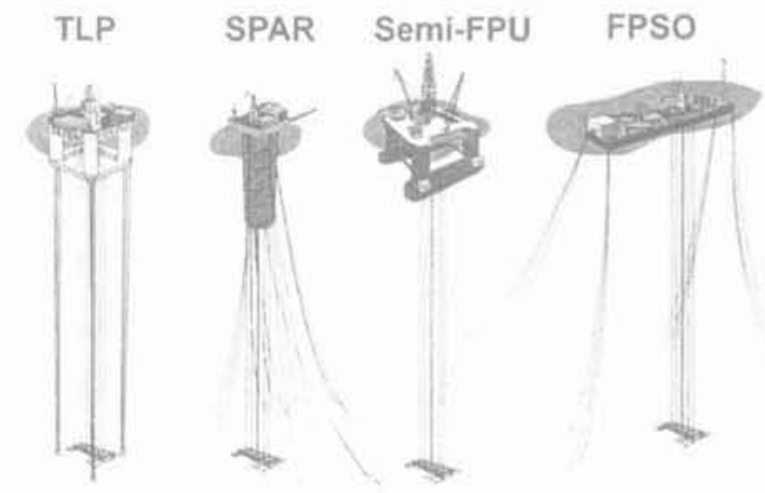

FIG. 1 Structures pétrolières flottantes en mer profonde.

Floating oil production structures for deep water sites.

Pour ces structures pétrolières flottantes en mer profonde, les ancrages par « caissons à succion $\gg$ font l'objet d'un vif intérêt dans l'industrie pétrolière, notamment du fait : (1) de leur plus grande fiabilité par rapport aux ancres traditionnelles, (2) de leur capacité à reprendre des efforts inclinés avec des lignes d'amarrage tendues et (3) des économies engendrées par la réduction des temps et des moyens d'installation requis. Dans la littérature anglo-saxonne, ces caissons à succion, en acier ou béton et mis en place dans le sol en créant une dépression à l'intérieur du caisson par rapport à la pression hydrostatique ambiante sur le fond marin, sont généralement mentionnés sous les termes de " skirted foundations and anchors » (fondations et ancres à bêches/jupes), " bucket foundations » (fondations de type seau retourné), ou "suction anchors $\cdots$ (ancres à succion).

Après une première application pour l'ancrage d'un stockeur pétrolier (bouée pour FSO) sur le champ de Gorm au Danemark en 1981 [2], le concept de fondation et d'ancrage par caissons à succion a rencontré un regain d'intérêt depuis le début des années 90 . On peut notamment mentionner les applications pour les fondations et ancrages des structures pétrolières suivantes:

- En mer du Nord : les plates-formes à lignes tendues (TIP) de Snorre [3] et Heidrun (avec des caissons en béton), les plates-formes treillis fixes ( j jackets $\gg$ ) de Draupner (auparavant Europipe [4]) Sleipner et Alba, les plates-formes autoélévatrices ( jack-ups $n)$ de Sleipner et Yme [5], les unités flottantes de production et stockage (FPSO) de Yme, Schiehallion [6], Curlew [7], Asgard A et Jotun, ainsi que les plates-formes semisubmersibles de production de Njord, Visund [8] et Asgard B.

- Pour l'ancrage permanent d'unités de production et de stockage en mer profonde : la barge de production de Nkossa au Congo $[9,10]$, les FPSO de Aquila en mer Adriatique [7], de Lufeng en Chine et de Laminaria en Australie, ainsi que de Kuito et Girassol en Angola, les unités flottantes de production (FPU) de Marlim au Brésil [11], et la bouée SPAR de Diana dans le golfe du Mexique.

- Parmi les autres applications, on peut également citer: les fondations de structures de préforage comme celles de Snorre en Norvège [3] et de Nkossa au Congo [12] ou celles de petites structures de protection de têtes de puits sous-marines [13], l'ancrage temporaire d'appareils de forage d'exploration par grandes profondeurs d'eau dans le golfe du Mexique [14], les fondations permanentes de la structure support de la torche de Nemba au Cabinda ainsi que les fondations temporaires du « jacket $)$ de Mahogany à Trinidad [15], la fixation des extrémités d'une conduite sous-marine sur le champ de Cantarell au Mexique et l'ancrage de lignes de mouillage dans plusieurs ports en Hollande.

Ainsi, le système d'ancrage de la barge de Nkossa en 1995 a représenté la première application après l'ancrage du stockeur pétrolier de Gorm en 1981. Depuis, les applications de caissons à succion se sont multipliées pour l'ancrage permanent de structures pétrolières flottantes (voir le tableau I ci-dessous).

Les nombreuses applications résumées dans le tableau I démontrent la grande versatilité des caissons à succion, utilisés pour une large gamme de profondeur d'eau (40 à 1400 mètres) et dans tous les types de sol, depuis les sables denses et argiles raides de mer du Nord jusqu'aux argiles très molles du golfe du Mexique et des sites en mer profonde.

Le dimensionnement géotechnique des caissons à succion est basé sur l'expérience acquise avec les structures gravitaires de mer du Nord équipées de bèches ( skirts »), notamment Gullfaks (installation en 1989 par 220 m d'eau, avec des bêches de 22 mètres) 
TABLEAU Applications des caissons à succion pour l'ancrage permanent de structures flottantes hors TLP (tableau modifié d'après [16]).

\begin{tabular}{|c|c|c|c|}
\hline Champ et structure & Année & $\begin{array}{l}\text { Prof. } \\
\text { (m) }\end{array}$ & $\begin{array}{l}\text { Dimensions. } \\
\text { Diam x Pén. }\end{array}$ \\
\hline $\begin{array}{l}\text { Gorm (FSO Shell) } \\
\text { Nkossa (Barge Elf) } \\
\text { Yme (FPSO Statoil) } \\
\text { Norne (FPSO Statoil) } \\
\text { Njord (Semi-FPU Hydro) } \\
\text { Curlew (FPU Shell) } \\
\text { Marlim P-19 et P-26 } \\
\text { (Semis-FPU Petrobras) } \\
\text { Schiehallion (FPSO BP) } \\
\text { Visund (Semi-FPU Hydro) } \\
\text { Lufeng (FPSO Statoil) } \\
\text { Aquila (FPSO Agip) } \\
\text { Laminaria } \\
\text { (FPSO Woodside) } \\
\text { Marlim P33-P35 } \\
\text { (FPSO Petrobras) } \\
\text { Jotun (FPSO Esso) } \\
\text { Asgard A (FPSO Statoil) } \\
\text { Asgard B et C (FPU Statoil) } \\
\text { Troll C (Semi-FPU Hydro) } \\
\text { Kuito (FPSO Chevron) } \\
\text { Diana (SPAR Exxon) } \\
\text { Barracuda P34? } \\
\text { (FPSO Petrobras) } \\
\text { Espadarte P48? } \\
\text { (FPSO Petrobras) }\end{array}$ & $\begin{array}{l}1996 \\
1997\end{array}$ & $\begin{array}{r}40 \\
200 \\
100 \\
350 \\
330 \\
90 \\
700 \\
1000 \\
350 \\
350 \\
330 \\
850 \\
400 \\
\\
780 \\
850 \\
130 \\
320 \\
300 \\
330 \\
400 \\
1400 \\
850 \\
\\
950 \\
\end{array}$ & $\begin{array}{c}3,5 \mathrm{~m} \times 9 \mathrm{~m} \\
4,5-5 \mathrm{~m} \times 12 \mathrm{~m} \\
5 \mathrm{~m} \times 7 \mathrm{~m} \\
5 \mathrm{~m} \times 10 \mathrm{~m} \\
5 \mathrm{~m} \times 7-10 \mathrm{~m} \\
5-7 \mathrm{~m} \times 9-12 \mathrm{~m} \\
4,7 \mathrm{~m} \times 13 \mathrm{~m} \\
6,5 \mathrm{~m} \times 12 \mathrm{~m} \\
5 \mathrm{~m} \times 11 \mathrm{~m} \\
5 \mathrm{~m} \times 10 \mathrm{~m} \\
4,5-5 \mathrm{~m} \times 16 \mathrm{~m} \\
5,5 \mathrm{~m} \times 13 \mathrm{~m} \\
4,7 \mathrm{~m} \times 17 \mathrm{~m} \\
? \\
5 \mathrm{~m} \times 11 \mathrm{~m} \\
5 \mathrm{~m} \times 11 \mathrm{~m} \\
5 \mathrm{~m} \times 15 \mathrm{~m} \\
3,5 \mathrm{~m} \times 13 \mathrm{~m} \\
6,5 \mathrm{~m} \times 30 \mathrm{~m} \\
? \\
?\end{array}$ \\
\hline Girassol (FPSO Elf) & 2001 & 1400 & $4,5 \mathrm{~m} \times 16,5 \mathrm{~m}$ \\
\hline
\end{tabular}

et Troll (plate-forme installée en 1995 par $300 \mathrm{~m}$ d'eau, avec des bêches de 36 mètres), complétée par des projets de $R \& D$ [17], la réalisation d'essais en modèles réduits sur site à terre [18-20] ou en centrifugeuse [21,22], ainsi que des essais en vraie grandeur sur site en mer [23]. Au bilan, et en moins de cinq ans, ce nouveau concept de fondation et d'ancrage a donc acquis un niveau de confiance suffisant pour être maintenant appliqué aux champs pétroliers en mer profonde où une fiabilité maximale est recherchée.

Enfin, parmi les autres types d'ancrages considérés, on peut noter que :

- les pieux d'ancrage battus, utilisés notamment pour plusieurs structures par grand fond dans le golfe du Mexique (dans des profondeurs d'eau comprises entre 500 et 1200 mètres pour les TLP des champs de Auger, Mars, Ram-Powell, Ursa, Morpeth et Marlin, comme pour les bouées SPAR de Neptune et Genesis par 550 et 900 mètres d'eau), sont limités aux capacités des systèmes de battage hydraulique sous-marin, soit actuellement environ 1500 mètres de profondeur d'eau :

- les pieux d'ancrage forés cimentés, bien qu'utilisés au Brésil par environ 800 mètres d'eau pour les FPSO P 33 et $\mathrm{P}-35$ sur le champ de Marlim, sont généralement considérés comme trop coûteux du fait des tarifs journaliers des appareils de forage :

- face aux caissons à succion, seul le nouveau concept d'ancre à capacité de chargement vertical ( V VLA » Vertically Loaded Anchor) semble avoir trouvé un marché en mer profonde, notamment pour les systèmes d'ancrage temporaires [24-26]. Des ancres VLA installées par l'intermédiaire d'un caisson à succion
( SEPLA» Suction Embedded Plate Anchor et «SEA» Suction Embedded Anchor) sont également en cours de développement [27,28];

- enfin, des ancres classiques ont été utilisées pour le FPSO- П du champ de Marlim Sud au Brésil par 1400 mètres de profondeur d'eau, ceci représentant probablement une des dernières applications des ancres conventionnelles pour de telles profondeurs. A noter que PETROBRAS prévoit de déplacer le FPSO-II sur un nouveau site par 1200 mètres de profondeur d'eau courant 1999, les ancres devant être remplacées par des VLA pour réduire la zone d'emprise du système d'ancrage sur le fond [29].

\section{2}

\section{Expérience du champ de Nkossa (200 mètres de profondeur d'eau au Congo)}

Le dimensionnement des caissons d'ancrage de la barge de production de Nkossa est résumé dans cette publication, en se limitant aux aspects généraux et aux opérations d'installation. Le dimensionnement géotechnique des caissons a été réalisé par le « Norwegian Geotechnical Institute n. (NGI, Oslo) pour " Single Buoy Moorings) (SBM, Monaco) en charge de la fourniture et de la pose du système d'ancrage, avec classification par le Bureau Veritas tel que requis par ELF Congo. Le principe du dimensionnement géotechnique des caissons à succion est détaillé dans les publications NGI [17] et [20]. Le comportement des caissons, enregistré en cours d'installation sur le site de Nkossa est décrit dans la présente publication, en mettant l'accent sur les enseignements applicables aux grands fonds et notamment au projet Girassol par 1400 mètres de profondeur d'eau en Angola.

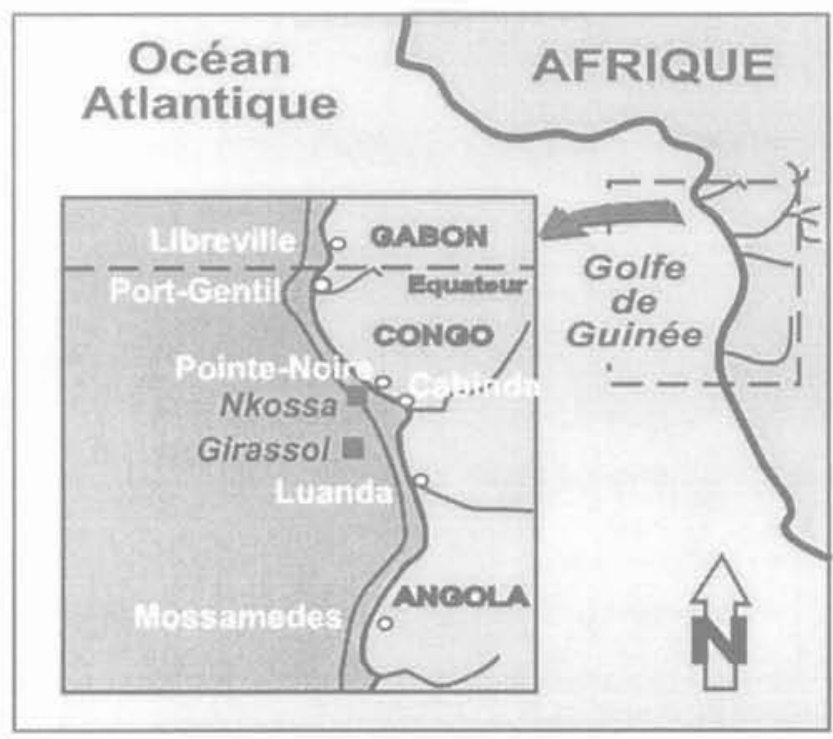

FIG. 2 Carte de situation générale.

General location map. 


\section{Caractéristiques du site et du système d'ancrage}

Le champ pétrolier de Nkossa, opéré par ELF Congo dans le golfe de Guinée, est situé par une profondeur d'eau comprise entre 150 et 300 mètres (Fig. 2). Ce développement comprend deux plates-formes fixes en acier (installées début 1995), avec forage assisté par appareil semi-submersible flottant, une barge de production et d'habitation (connectée aux plates-formes début 1996 pour la mise en production du champ), et deux unités flottantes de stockage et transfert, un pour I'huile et l'autre pour le gaz de pétrole liquéfié ( ( GPL »).

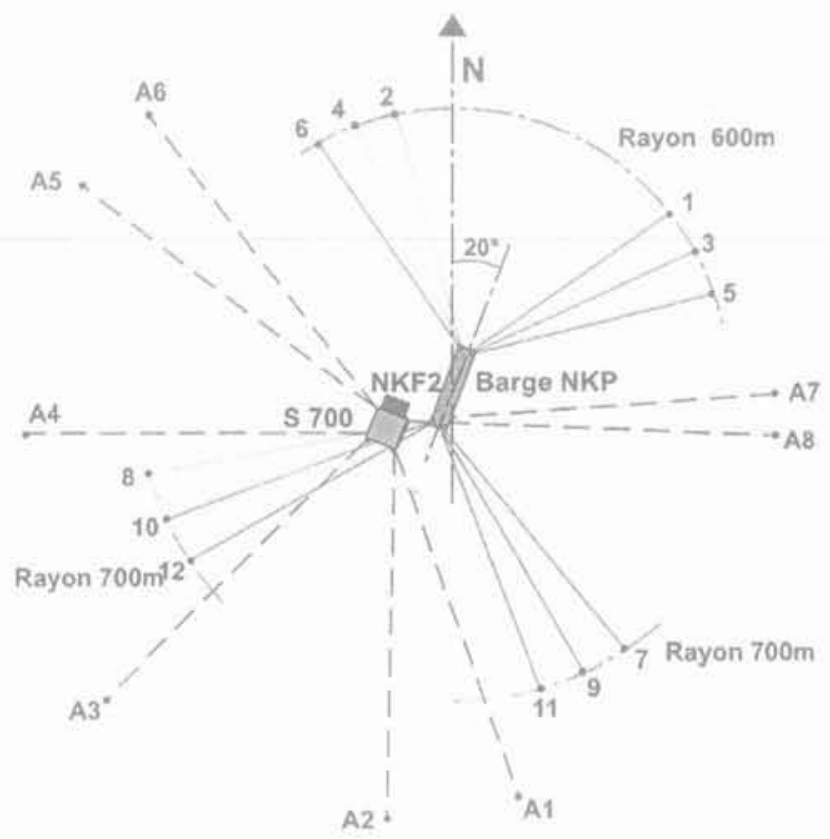

FIG.3 Nkossa - Systèmes d'ancrage de la barge NKP et de I'appareil de forage SEDCO S700.

Nkossa - Anchoring systems of the NKP barge and drilling rig SEDCO S700.

La barge de production et d'habitation de Nkossa a été prévue pour supporter 33000 tonnes d'équipements et pour loger jusqu'à 160 personnes. Sur le site, la barge NKP, positionnée à proximité de la plate-forme NKF2 et de l'appareil de forage SEDCO «S700 », est ancrée avec douze lignes d'amarrage composées de chaines de $11,4 \mathrm{~cm}$ de diamètre et réparties par groupes de trois. La gestion des risques d'interférence, possibles entre le système d'ancrage de la barge (12lignes) et celui de lappareil de forage ( 8 lignes), compliquée par la présence de la plate-forme voisine et des conduites sous-marines sur le fond, a été une contrainte non négligeable du schéma de développement du champ (Fig. 3).

Le champ de Nkossa est situé légèrement au Nord du canyon du fleuve Congo, à environ 60 kilomètres au large de la ville de Pointe Noire. A la limite du talus continental, le fond marin présente une pente moyenne d'environ $3 \%$. Des sondages géotechniques ont été réalisés sur chaque emplacement de groupe d'ancres, incluant des essais in situ au pénétromètre statique (essais PCPT au piézocône) et des

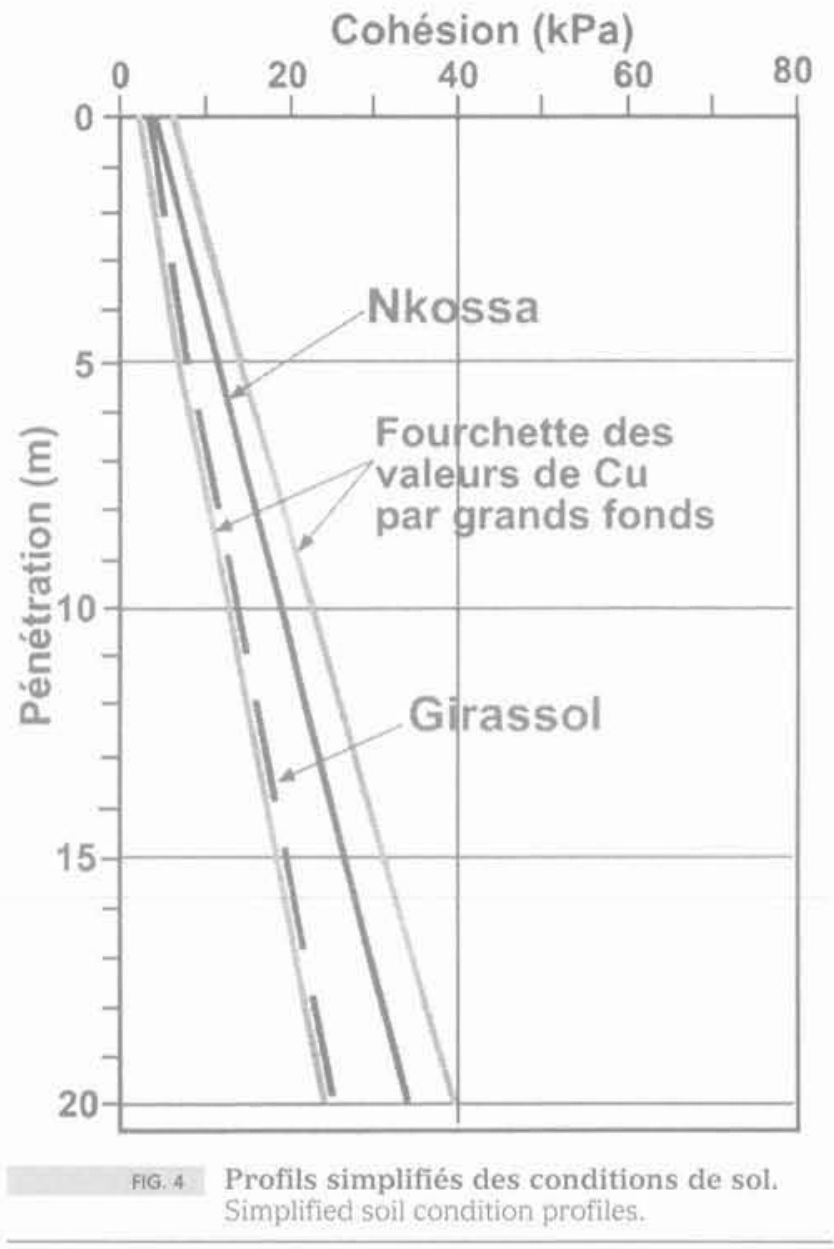

prélèvements d'échantillons pour essais de laboratoire. Dans une profondeur d'eau comprise entre 150 et 200 mètres sur la zone d'emprise des ancrages de la barge, une couche d'argile sableuse est présente sur le fond marin, avec une épaisseur variant de 2 à 5 mètres. Sous cette formation superficielle, les conditions de sol sont composées d'argile plastique normalement consolidée, avec une résistance au cisaillement non drainé croissant plus ou moins linéairement avec la pénétration sous le fond de mer. Le profil de résistance au cisaillement non drainé de l'argile, basé sur les résultats d'essais triaxiaux UU, est résumé à la figure 4 pour les 20 premiers mètres de sédiments.

En phase d'avant-projet, une comparaison des différents types d'ancrages possibles a été réalisée, incluant les ancres conventionnelles, les pieux d'ancrage battus et les caissons à succion [9]. Les résultats de cette étude sont résumés par la figure 5. La solution par caissons à succion a été préférée au cas de base avec des ancres conventionnelles pour les deux raisons suivantes : (1) une emprise réduite sur le fond marin avec un plus faible rayon d'ancrage du à la possibilité de reprendre des efforts inclinés (sur les douze lignes d'amarrage de la barge, une économie de 3000 mètres de chaîne, soit 800 tonnes d'acier, a été possible) et (2) une réduction des temps d'installation, notamment du fait qu'il n'était pas besoin de réaliser des essais de prétensionnement sur le site. Par comparaison avec les pieux d'ancrage battus, la mobilisation d'une barge grue de forte capacité, nécessaire pour la manipulation des pieux et d'un marteau hydraulique sous-marin, a été évitée. Au bilan, la réduction de coût liée à la solution par caissons à succion a été estimée 
égale à $20 \%$ du coût global de fabrication et d'installation du système d'ancrage de la barge, soit une économie de l'ordre de $20 \mathrm{MF}$.

Le dimensionnement du système d'ancrage de la barge a été réalisé pour satisfaire les conditions requises pour la durée de vie de trente ans du champ de Nkossa et en accord général avec la procédure du document API RP 2FP1 [30], Les coefficients de sécurité globaux appliqués étaient les suivants :

- pour les lignes $n^{\circ} 1,3,5,7,9$ et 11 (dont la rupture entraînerait un déplacement de la barge vers la plateforme NKF2, voir la figure 3) : 2,20 et 1,75 en condition " système intact ) et ( système avec une ligne cassée », respectivement :

- pour les lignes $n^{\circ} 2,4,6,8,10$ et 12 (dont la rupture entraînerait un déplacement de la barge à l'écart de la plate-forme NKF2, voir la figure 3) : 1,75 et 1,25 en condition « système intact ») et « système avec une ligne cassée 0 , respectivement.

Finalement, deux dimensions de caissons d'ancrage ont été adoptées pour les douze lignes d'amarrage de la barge: (1) diamètre $4,5 \mathrm{~m}$ et pénétration $11,8 \mathrm{~m}$, correspondant à une capacité ultime de 4,85 MN pour huit lignes (poids des caissons égal à 41 tonnes avec une épaisseur d'acier de $15 \mathrm{~mm}$, y compris 10,5 tonnes pour le capot amovible) et (2) diamètre $5 \mathrm{~m}$ et pénétration $12,5 \mathrm{~m}$ (figure 6), correspondant à une capacité ultime égale à $5,92 \mathrm{MN}$ pour quatre lignes supportant des efforts plus importants (poids des caissons égal à 47 tonnes avec le capot amovible).

Deux points particuliers peuvent être soulignés: - Pour les deux types de caissons, le point d'amarrage de la ligne d'ancrage est situé à environ $7,5 \mathrm{~m}$ sous le fond marin, de manière à minimiser le moment de renversement. En recherchant le point d'attache correspondant à une rotation nulle, la capacité portante ultime du caisson est optimisée en imposant une cinématique de translation latérale. Pour des conditions identiques, la connexion de la chaîne au niveau du fond marin entrainerait une division par deux de la capacité portante ultime des caissons (voir [9] et [18]).

- Du fait des efforts d'ancrage essentiellement horizontaux, la fermeture des caissons n'était pas requise et un capot amovible a été utilisé. Adaptable aux deux types de caissons et permettant un gain supplémentaire en poids d'acier, celui-ci n'a été utilisé que pour la phase de pénétration dans le sol par dépression.

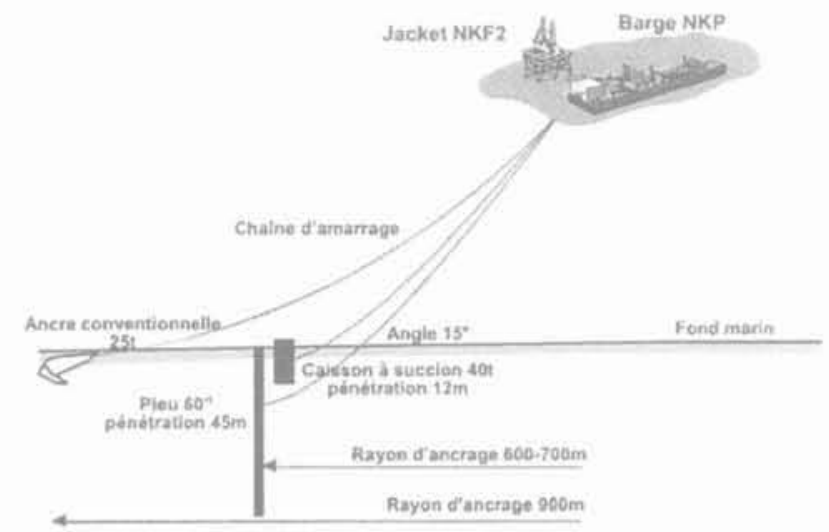

Fig.5 Nkossa - Comparaison des systèmes d'ancrage considérés.

Nkossa - Comparison of the anchoring systems considered.

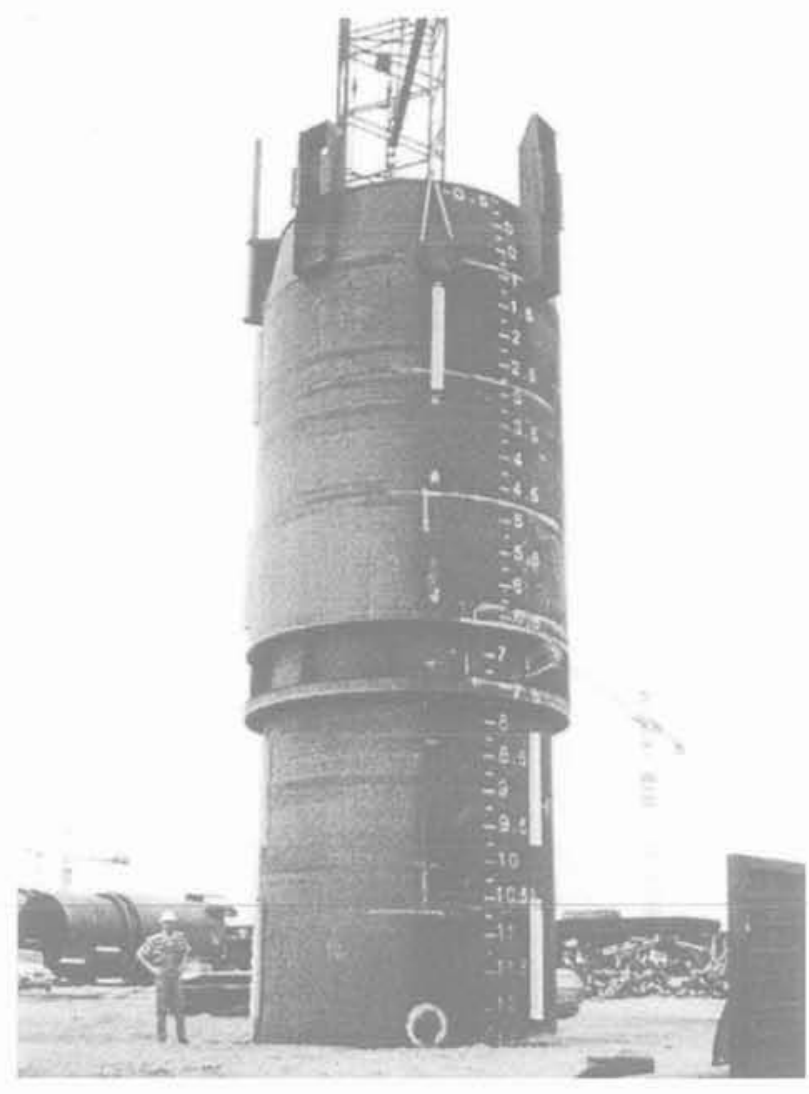

FG. 6 Nkossa-Caisson à succion pour la barge. Nkossa - Suction caisson for the barge.

Enfin, étant donné la capacité des caissons à reprendre des efforts verticaux, le rayon d'ancrage a pu être réduit jusqu'à autoriser un soulèvement de la ligne d'ancrage avec un angle de l'ordre de $15^{\circ}$ par rapport au fond marin dans le cas des efforts extrêmes (voir la figure 5). A l'époque du dimensionnement du système d'ancrage de la barge Nkossa, ceci représentait une déviation par rapport à l'API RP 2FPI où il est considéré que les ancres conventionnelles ne peuvent reprendre que des efforts horizontaux. La possibilité de reprendre des efforts inclinés a ensuite été reconnue par l'API RP 2SK, tout en limitant l'angle d'inclinaison de la ligne d'ancrage à $5^{\circ}$ et $10^{\circ}$ en condition de chargement extrême pour les cas "système intact ) et " système avec une ligne cassée ), respectivement (voir [30] et [31]).

\section{Procédure et prévision d'installation}

Compte tenu de la relativement faible distance entre la terre et le site de Nkossa $(4,5$ heures de navigation), un seul navire a été utilisé pour le transport et l'installation des caissons. Le " Dynamic Installer n, navire à positionnement dynamique opéré par SBM, est équipé d'une grue de 100 tonnes de capacité de levage et d'un véhicule télé-opéré pour l'observation sous-marine (ROV). Transportés un par un en position verticale sur le pont du navire, les caissons ont été mis à l'eau par la grue du bord, après connexion de la chaine et vérification du système de pompage et d'instrumentation. 


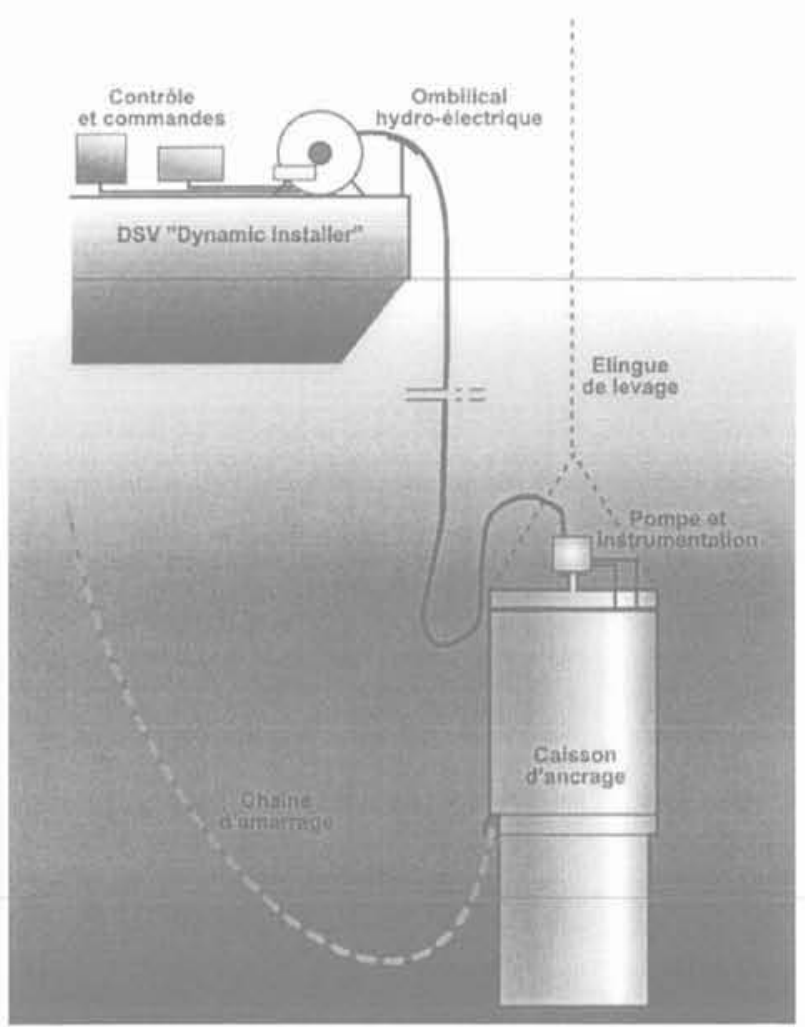

FIG, 7 Nkossa - Mise à l'eau du caisson avec le système de pompage et d'instrumentation. Nkossa-Over-boarding of the caisson with the pumping and monitoring system.

Le système de pompage et d'instrumentation, développé par le NGI, était positionnné sur un perçage de diamètre $0,51 \mathrm{~m}$ au centre du capot amovible du caisson. Ce système, relié au navire par un ombilical hydroélectrique pour les commandes et la transmission en temps réel des données d'instrumentation (Fig. 7), comprenait l'équipement suivant :

- balise acoustique et gyro-compas pour le positionnement précis du caisson et le contrôle de son orientation sur le fond marin :

- capteur de pression totale pour la mesure de l'immersion du caisson :

- pompe centrifuge hydraulique, utilisée pour l'évacuation de Yeau lors de la pénétration du caisson dans le sol. En cours de pénétration par succion, le contrôle de la dépression à l'intérieur du caisson était assuré par le contrôle du débit de la pompe évacuant l'eau du caisson:

- inclinomètres pour la mesure de l'inclinaison du caisson selon deux directions au moment de la pose sur le fond et en cours de pénétration dans le sol ;

- écho-sondeurs, un à l'extérieur et l'autre à l'intérieur du caisson, pour la mesure de la pénétration (en plus des marquages sur le caisson suivis par la caméra du ROV sur le fond) et de l'éventuel soulèvement du sol à l'intérieur du caisson en cours de pénétration par succion: - capteur de pression différentielle pour la mesure de la dépression (donc de l'effort de pénétration par succion) créée par le pompage de l'eau emprisonnée à l'intérieur du caisson.
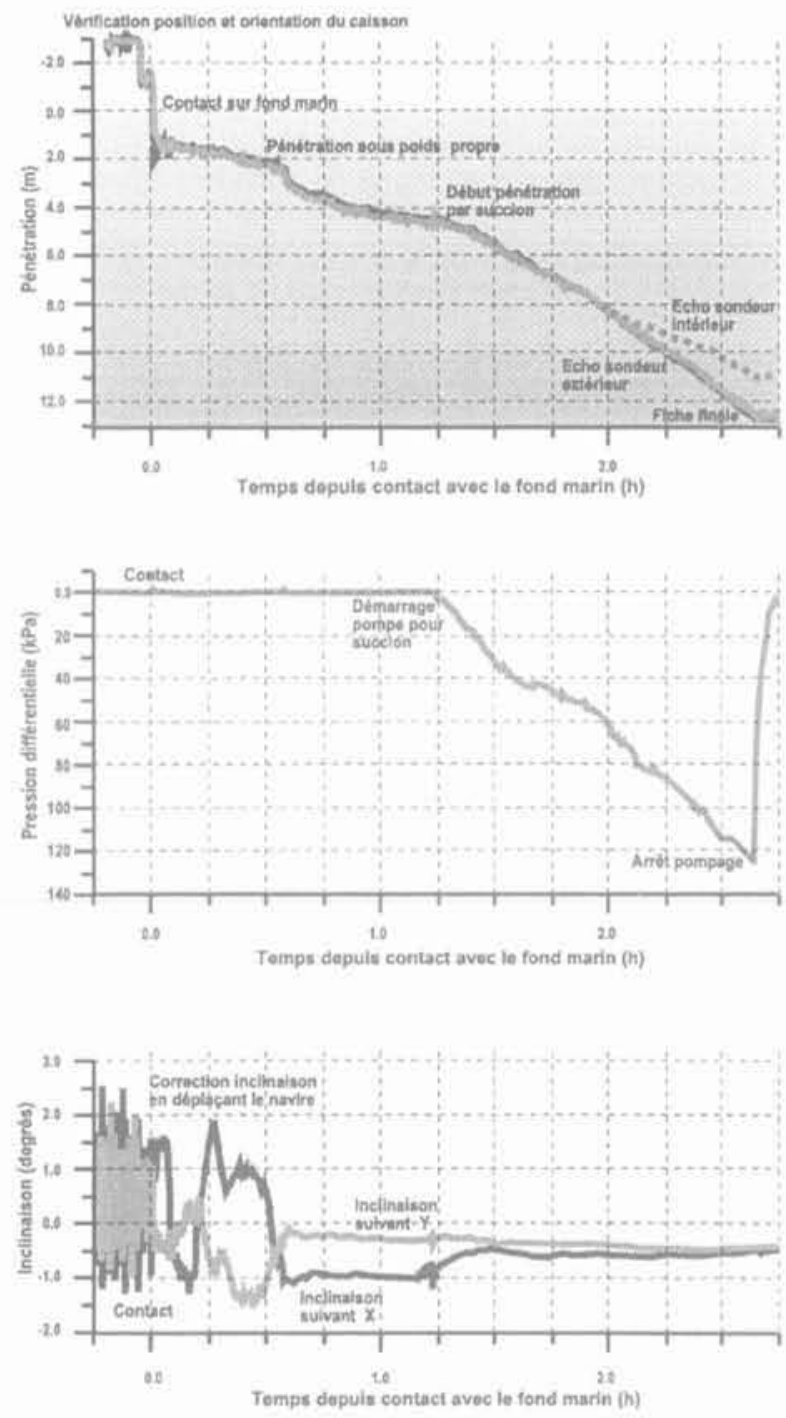

FIG. 8 Nkossa - Phases d'installation d'un caisson par succion.

Nkossa - Installation phases of a suction caisson.

La prévision d'installation est résumée à la figure 9 , où sont donnés, d'une part, le faisceau prévisionnel de dépression en fonction de la pénétration des caissons dans le sol et, d'autre part, la valeur « critique » de dépression correspondant à la rupture du sol par soulèvement à l'intérieur du caisson. Ce soulèvement du "bouchon » de sol pouvant entrainer un refus de pénétration, la succion créée par le pompage en cours de pénétration doit être maintenue en deçà de cette valeur dite « critique ». La comparaison avec les résultats obtenus en cours de pénétration est faite ci-dessous.

\section{3}

\section{Résultats des opérations d'installation}

Les résultats obtenus en cours d'installation d'un caisson sont illustrés par la figure 8 où les différentes étapes de mise en place sont données en fonction du temps, en présentant : (1) la pénétration du caisson dans le sol (graphe supérieur, noter que les deux échos-sondeurs donnent des valeurs de pénétration légèrement différentes), (2) l'évolution de la pression différentielle à l'intérieur du caisson (graphe central) et 


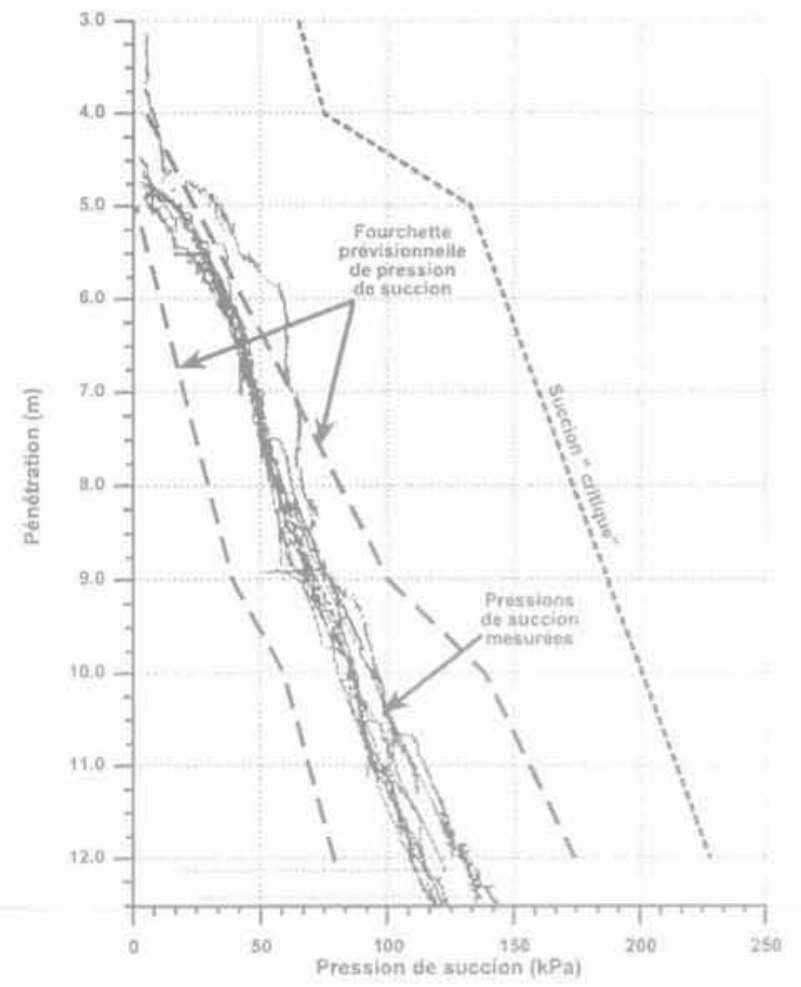

FIG. 9 Nkossa - Comparaison entre prévision et résultats d'installation.

Nkossa - Comparison between prediction and resuits of installation.

(3) l'évolution de l'inclinaison du caisson (graphe inférieur). Les résultats présentés à la figure 8 sont représentatifs du comportement moyen obtenu pour les douze caissons d'ancrage de la barge de Nkossa.

Lors des différentes phases de mise en place d'un caisson, on relève les points particuliers suivants:

- Pénétration : Après vérification de la position et de l'orientation du caisson à l'approche du fond marin, le caisson s'enfonce dans l'argile molle sous poids propre. Une pénétration de l'ordre de $4,5 \mathrm{~m}$ est obtenue avant de démarrer la phase suivante de pénétration par succion (voir également la figure 9 résumant les résultats obtenus pour les douze caissons). Tous les caissons ont été amenés à leur fiche de projet avec une vitesse de pénétration variant entre 3,6 et $10,5 \mathrm{~m} / \mathrm{h}$, la vitesse moyenne de $6,5 \mathrm{~m} / \mathrm{h}$ correspondant à un débit moyen de la pompe hydraulique de $110 \mathrm{~m}^{3} / \mathrm{h}$.

- Pression différentielle : La dépression (ou succion) maximale requise pour amener les caissons à leur fiche de projet a été comprise entre - 115 et - $145 \mathrm{kPa}$, avec une valeur moyenne de $-125 \mathrm{kPa}$. La figure 9 montre que ces valeurs étaient en très bon accord avec le faisceau prévisionnel et que la marge de sécurité vis-à-vis de la valeur « critique » était importante. Dans le cas de Nkossa, on souligne que la dépression était uniquement requise pour la phase de pénétration, la pression hydrostatique étant rétablie à l'intérieur des caissons en fin de pénétration par l'arrêt de la pompe hydraulique avant de retirer le capot amovible portant le système de pompage et d'instrumentation.

- Inclinaison : A l'approche du fond de mer, et du fait des mouvements du navire dus à la houle, I'inclinaison du caisson variait de façon cyclique de \pm 1 à $2^{\circ}$, le contact avec le fond étant clairement mis en évidence par l'arrêt immédiat de ces mouvements. En début de pénétration sous poids propre, l'inclinaison évoluant rapidement dans les deux directions X (vers la chaîne d'amarrage) et Y (à $90^{\circ}$ de la chaine), elle a été corrigée en agissant sur l'effort de tension dans la chaîne d'amarrage (retenue sur le pont du navire). Cette solution s'est révélée très efficace, le caisson étant quasiment parfaitement vertical en fin d'installation. Tous les caissons ont été installés avec une inclinaison inférieure à la tolérance maximale, fixée à $\pm 10^{\circ}$ après avoir vérifié l'incidence d'une telle inclinaison sur la capacité portante ultime des caissons.

\section{3}

\section{Application au champ de Girassol (1400 mètres de profondeur d'eau en Angola)}

Le champ pétrolier de Girassol, opéré par EL F Exploration Angola, est situé à environ 200 kilomètres au large de Luanda par 1400 métres de profondeur d'eau. Le schéma de développement prévoit une unité flottante de production et stockage (FPSO) avec une quarantaine de puits sous-marins. MPG (" Mare Profundo Girassol), un groupement entre EIPM et Bouygues Offshorej est en charge de la fourniture du FPSO dont les dimensions en font le plus grand au monde : $300 \mathrm{~m}$ de long et $60 \mathrm{~m}$ de large, $2000000 \mathrm{bbl}\left(320000 \mathrm{~m}^{3}\right)$ de volume de stockage disponible et une capacité de traitement de $200000 \mathrm{bbl} /$ jour (environ $32000 \mathrm{~m}^{3} / \mathrm{j}$ our). La durée de vie estimée du champ est de vingt ans.

\section{1}

\section{Enseignements tirés de l'expérience Nkossa}

Les principaux enseignements tirés de l'expérience Nkossa et applicables aux grandes profondeurs d'eau sont les suivants :

- la pénétration de caissons par succion dans des argiles molles ne pose pas de problème particulier. Le comportement observé sur Nkossa a été confirmé par les expériences suivantes, notamment sur les sites de Aquila [7] et Marlim [11] dans des profondeurs d'eau comprises entre 700 et 1000 mètres (en attendant Diana, fin 1999). Il est maintenant considéré que la pénétration ou le retrait d'un caisson est possible jusqu'à un élancement hauteur/diamètre de l'ordre de 10 pour l'enfoncement par succion et 7 pour le retrait par surpression [16] ;

- un système d'instrumentation est indispensable. notamment pour contrôler le comportement du caisson en temps réel dès la phase initiale de contact avec le fond marin. En mer profonde, et dans le but d'éviter l'utilisation d'un ombilical entre la surface et le fond, le système de pompage et d'instrumentation peut ètre porté par un véhicule télé-opéré (ROV). Cela a déjà été réalisé sur plusieurs sites (voir [7, 11, 14, 23]) et des équipements sont disponibles avec une capacité de 1500 à 2000 mètres de profondeur d'eau :

- en cours d'installation, la correction de l'orientation du caisson est plus difficile que celle de son inclinaison. Dans le cas de Nkossa, la tolérance d'orientation était de $\pm 8^{\circ}$ et était liée à des limites de contrainte structurelle dans la zone de connexion de la chaîne. Il faut souligner que des tolérances sêvères peuvent avoir une 
incidence non négligeable sur le coût d'un système d'ancrage, notamment avec le risque d'augmenter le temps d'installation sur site (avec la répétition éventuelle d'opérations en mer pour poser les caissons selon les tolérances requises), ainsi que le coût des systèmes d'instrumentation nécessaires (avec également le besoin de prévoir une redondance des équipements). Pour pallier a ces problèmes, il est recommandé : (1) d'étudier la conception du point de connexion de la chaine pour pouvoir accepter des tolérances d'orientation plus larges et (2) de prendre en compte la baisse de capacité liée à une éventuelle inclinaison dans le dimensionnement des caissons ;

- pour chaque ligne d'amarrage de la barge Nkossa par 200 mètres de profondeur d'eau, le temps d'installation moyen a été égal à 18 heures (hors temps de navigation entre le port et le site), le temps de pose du caisson luimême ne représentant que 2 à 4 heures (voir figure 8). Dans la mesure où ce temps incluait un certain apprentissage pour une " première » pour SBM et NGI en 1995, on considère que l'installation d'un caisson avec sa ligne devrait étre faisable en 24 et 30 heures environ dans 1000 et 1500 mètres d'eat., respectivement.

\section{2}

\section{Installation sur Girassol}

Comme dans le cas de Nkossa, le dimensionnement géotechnique des caissons d'ancrage pour Girassol est réalisé par le NGI et fait l'objet d'une classification par le Bureau Veritas. Le profil de sol simplifié, composé essentiellement d'argile molle normalement consolidée, est résumée à la figure 4. Les dimensions générales des caissons d'ancrage sont données au tableaul.

Le système d'ancrage du FPSO de Girassol, composé de seize lignes réparties par groupes de quatre, doit être préinstallé début 2001, avant connexion à l'arrivée du navire pour la mise en production du champ courant 2001. Les caissons d'ancrage sont dimensionnés pour un effort ultime de $6,89 \mathrm{MN}$ avec un angle d'inclinaison de $25^{\circ}$ en concition "système avec une ligne cassée » (correspondant à un effort au caisson de $4,59 \mathrm{MN}$ avec un coefficient de sécurité global de 1,5). Les seize caissons et lignes d'amarrage, assemblés à terre en Angola, seront transportés sur le site de Girassol à bord d'une barge. Les opérations d'installation seront ensuite réalisées par une barge grue ou par un navire d'assistance à positionnement dynamique, équipés d'une grue de levage ainsi que de deux véhicules télé-opérés sous-marins (un ROV d'observation et un ROV de travail).

Pour chaque ligne d'ancrage, la procédure générale d'installation, globalement similaire à celle utilisée sur Nkossa, devrait être la suivante :

- le caisson, connecté à la ligne d'amarrage, est mis à l'eau par la grue du navire;

- après immersion, le ROV de travail, équipé du système de pompage et d'instrumentation, est accosté sur le capot du caisson (capot fixe dans le cas de Girassol) ;

- descente du caisson sur le fond marin et pénétration sous poids propre avec évacuation de l'eau emprisonnée dans le caisson par la pompe et des évents sur le capot:

- fermeture des évents avant démarrage de la phase de pénétration par succion. Le suivi est assuré par le ROV d'observation sur le fond de mer, ainsi que par le système d'instrumentation commandé depuis le navire à la surface :

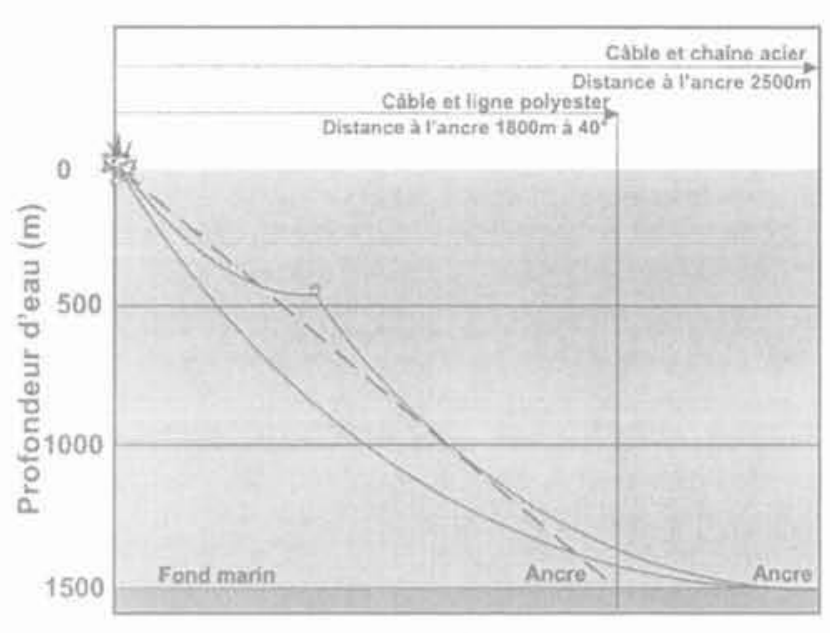

FG.10 Girassol - Schéma de principe d'un système d'ancrage à lignes tendues par rapport à un système conventionnel.

Girassol - Principle of a taut mooring system compared to a conventional system.

- après obtention de la fiche de projet, le système de pompage est arrêté et le ROV de travail est déconnecté du caisson pour être ramené à la surface :

- pose de la ligne d'ancrage sur le fond où elle est abandonnée jusqu'à l'arrivée et la connexion du FPSO environ trois mois plus tard.

Concernant le champ de Girassol, on peut enfin mentionner qu'un deuxième système d'ancrage avec caissons à succion ( 9 caissons de diamètre $5 \mathrm{~m}$ et de hauteur $15 \mathrm{~m}$ à fournir par SBM) doit également être réalisé pour une bouée servant à l'amarrage et au chargement des navires pétroliers ( (ankers $)$ ) venant s'approvisionner en huile sur le champ. Par rapport à celui du FPSO, ce système d'ancrage présentera la particularité d'être équipé de lignes tendues en fibres synthétiques, permettant de réduire son emprise sur le fond marin. Le schéma de principe d'un tel système d'ancrage est résumé à la figure 10: avec un angle de l'ordre de $40^{\circ}$ par rapport au fond marin, la longueur des lignes d'ancrage est d'environ 1800 mètres, ce qui représente une économie importante vis-à-vis du cas de base avec des ancres conventionnelles où une longueur minimale de 2500 mètres serait requise pour maintenir un effort horizontal à l'ancre.

\section{4}

\section{Conclusion}

Le Groupe ELF, avec l'application pour la barge de Nkossa réalisée en 1995 avec le soutien de SBM et NGL a eu un rôle pionnier dans le développement des caissons à succion. Depuis, le vif intérêt suscité par ce nouveau concept d'ancrage des structures pétrolières flottantes est clairement mis en évidence par le nombre croissant d'applications dans le monde entier. En plus d'une grande versatilité permettant de s'adapter à une large gamme de profondeur d'eau et à tous les types de sols, cet intérêt provient notamment: (1) d'une meilleure fiabilité par rapport aux ancres conventionnelles, (2) de la capacité à reprendre des efforts inclinés, avec la possibilité de réduire le rayon d'ancrage dans le cas de lignes d'amarrage tendues, et (3) de la réduction des coûts de mise en place, du fait de moyens et d'un temps d'installation réduits sur site. 
Sur le site de Nkossa, avec des conditions de sol composées d'argiles molles normalement consolidées représentatives des sites en mer profonde, une expérience et un savoir-faire précieux ont été acquis pour l'application de ce nouveau concept d'ancrage aux sites par grands fonds. A cette occasion, le parfait accord entre la prévision et les résultats d'installation, contrôlés en temps réel sur le site par un système d'instrumentation, a également permis de démontrer la fiabilité de la procédure de mise en place de ces caissons par dépression.

Début 2001, le champ de Girassol, par 1400 mètres de profondeur d'eau en Angola, verra une nouvelle application des caissons à succion pour l'ancrage d'un navire de stoc- kage et de production (FPSO) et d'une bouée de chargement. Après l'ancrage des FPU P-19 et P-26 sur le champ de Marlim au large du Brésil et de la SPAR de Diana dans le golfe du Mexique, l'utilisation de lignes d'amarrage tendues en fibres synthétiques associées à des caissons à succion pour cette bouée de chargement sur Girassol, marque le lancement d'une nouvelle génération d'ancrage pour l'ultra grand fond, au-delà de 1500 mètres de profondeur d'eau.

\section{REMERCIEMENTS}

L'auteur remercie EIF Congo, ELF Exploration Angola et ELF Exploration Production pour la permission de publier cet article.

\section{Bibliographie}

(1) Lacasse S. - Geotechnical contributions to offshore development. $31^{\circ}$ conférence OTC (a Offshore Technology Conf. v), Houston, mai 1999, publication OTC 10822.

[2] Senpere D., Auvergne G.A. - Suction anchor piles: a proven alternative to driving or drilling. 14c conférence OTC ( Offshore Technology Conf, $»$ ), Houston, mai 1982, publication OTC 4206

[3] Christophersen H.P., Bysveen S. Stove O.J. - Innovative foundation systems selected for the Snorre field development. $6^{e}$ conférence BOSS (« Behaviour of Off-Shore Structures n), Londres, juillet 1992, BPP Technical Services Ltd, 1, p. 81-94.

[4] Tjelta T.I. - Geotechnical experience from the installation of the Europipe jacket with bucket foundations. $27^{\mathrm{e}}$ conférence OTC ( Offshore Technology Conf. $n$ ). Houston, mai 1995, publication OTC 7795 .

[5] Eide A. Tuen K.A., Baerheim M. - The Yme jack-up with skirt foundation. $28^{\circ}$ conférence OTC \& Offshore Technology Conf. 11), Houston, mai 1996, publjcation OTC 8158

[6] Krudsen A. Pettiorew G. - Hiah capacity suction anchors for BP's Schielhallion EPSO. Conférence IBC, FPS 97 (œ Floating Production Systems). Londres, avril 1997.

[7] Alhayari S: - Innovative developments in suction pile technology. $30^{\circ}$ conférence OTC (« OIfshore Technology Conf. ») Houston, mai 1998, publication OTC 8236.

[8] Solhjell E. Sparrevik P. Haldorsen K. Karlsen V. - Comparison and back calculation of penetration resistance from suction anchor installation in soft to stiff clay at the Njord and Visund fields in the North Sea, Conference SUT ( « Society for Underwater Technolocry n) «New Frontiers in Offshore Site Investigation and Foundation Behaviour B, Londres, septembre 1998, p. 325-349.

[9] Colliat J.L.. Boisard P., Andersen K. Schroeder K. - Caisson foundations as alternative anchors for permanent mooring of a process barge offshore Congo $27^{\circ}$ conférence OTC la Offshore Technology Conf, n), Houston, mai 1995, publication OTC 7797

[10] Colliat J.L., Boisard P., Gramet J.C., Sparrevik P. - Geotechinical design and installation behaviour of suction anchor piles. Comparison with drag anchors. ge conférence BOSS (« Behaviour of OffShore Structures »), Delft, juillet 1997. Pergamon, 1, p. 133-147.

[11] Mello J.R.C. Moretti M.J.. Sparrevik. P.
Schröder K Hansen S B - P19 and P26 moorings at the Marlim field - The first permanent taut leg mooring with fibre rope and suction anchors. Conférence FPS 98 ( $w$ Floating Production Sys: tems w), Londres, 1998

[12] Jandinier R.C. Bouger Y.. Boisard P. Construction and installation of tem plates for deep waters in west Africa. $13^{e}$ conférence OMAE (* Offshore Mechanics and Arctic Engineering p), Houston, mars 1994, 1. p. 433-440.

[13] Guttormsen T.R. Wikdal J.A. - Foundation of the Tordis submudline silo. $7^{e}$ conférence BOSS (" Behaviour of Off. Shore Structures v), Boston, juillet 1994. Pergamon, 1. p. 189-203.

(14) El-Gharbawy S, Olson R.E., Scott S.A. Suction anchor installations for deep gulf of Mexico applications. $31^{\epsilon}$ conférence OTC fu Offshore Technology Conf. »). Houston, mai 1999, publication OTC 10992

[15] Green D.. Jeanjean P. - Innovative temporary foundation system for jacket installation and levelling. $9^{\circ}$ conférence ISOPE fu int. Serninar on Offshore and Polar Engineering). Brest, fuin 1999, 1. p. 631-638.

[16] Sparrevik P. Suction anchors - A versatile foundation concept finding its place in the offshore market. Conférence OMAE (i Offshore Mechanics and Arctic Engineering n), publication OMAE 98-3096, Lisbonne, juillet 1998.

[17] Andersen K.H., Jostad H.P. - Foundation design of skirted foundations and anchors in clay, $31^{\circ}$ conférence OTC (aOffshore Technolocy Conf, s), Houston, mai 1999, publication OTC 10824

[181 Keaveny J.M Hansen S.B. Madshus C., Dyvik R. - Horizontal capacity of large-scale model anchors, $13^{\circ}$ conférence ISSMFE fil Int, Society for Soil Mechanics and Foundation Engineering v), New Dehli, 1994, 2, p. $677-680$.

[19] Dyvik R. Andersen K.H. Borg HansenS., Christophersen H.P. - Field tests of anchors in clay. Part 1: Description. Journal of Geot. Eng. ASCE (a American Society of Civil Engineers p). 119, 10, octobre 1993, p. 1515-1531.

[20] Andersen K.H. Dvvik R. Schrö̀der K. Hansteen O.E. Bysveen S. - Field tests of anchors in clay, Part II: Predictions and interpretation. Journal of Geot. Eng. ASCE $(\alpha$ American Society of Civil Engineers s), 119, 10, octobre 1993, p. 1532-1549.

[21] Clukey E. C. Morrison M.1. Garnier I. Corté J.F. - The response of caissons in normally consolidated clays to cyclic TLP loading conditions. $27^{\circ}$ conférence
OTC (« Offshore Technology Conf. 1), Houston, mal 1995, publication OTC 7796

(22) Randoiph M.F., O'Neill M.P., Stewart D.P. - Performance of suction anchors in fine-arained calcareous soils. $30^{\circ}$ conférence OTC (a Offshore Technology Conf. n), Houston, mai 1998, publication OTC 8837

[23] Olberg T.S. Guttormsen T., Molland G. Andersen J. - Full scale field trial of taut leg mooring using fibre rope and suction anchor attached to a semi-submersible drilling unit. 29" conférence OTC f $($ Offshore Technology Conf, v), Houston, mai 1997, publication OTC 8357

[24] Agnevall T. - Installation and performance of P-27 Stevmanta VLA anchors Conférence llR « Moorings \& Anchors for Deep and Uitra Deep Water Fields x, Aberdeen, octobre 1998.

[25] Foxton P. - Latest developments for vertically loaded anchors. Confèrence IBR \& Mooring and Anchoring p, Aberdeen, juin 1997

26] Colliat J.L. Foulhoux L. - Suction piles versus drag anchors for deep water moorings. $8^{*}$ conférence ISOPE $(a$ int Seminar on Offshore and Polar Engineering n), Montréal, mai 1998, 2, p. 225 232

[27] Dove P. Treu H. Wilde B. - Suction embedied plate anchor (SEPLA). A new anchoring solution for ultra deep wate mooring 10* conférence DOT lu Deep Offshore Technology w) New Orleans, nov. 1998.

[28] Riemers M.E., Kirstein A.A. - Examining the development and capabilities of the alternative Suction Embedded Anchor (SEA) in deep and ultra deep water. Conference IIR a Moorings \& Anchors for Deep and Ultra Deep Water Fields »), Aberdeen, nov. 1999 (à publier).

[29] Barusco P.-Mooring and anchoring systems developed in Marlim field. 31 conférence OTC ( ( Offshore Technology Conf, p) Houston, mai 1999, publication OTC 10720

[30] American Petroleum Institute RP 2FP1. - Recommended practice for design. analysis and maintenance of moorings for floating production systems. $1^{\text {re }}$ édition. API, Washington DC, 1993.

[31] American Petroleum Institute RP 2SK - Recommended practice for design and analysis of stationkeeping systems for floating structures, $1^{\text {re }}$ édition, AP1. Washington DC. 1995. 